\title{
The PSU/TCfA search for planets around evolved stars. Stellar parameters and activity indicators of targets.
}

\author{
Andrzej Niedzielski ${ }^{1}{ }^{12}$, Grzegorz Nowak ${ }^{1}$ and Paweł Zieliński ${ }^{1}$ \\ ${ }^{1}$ Toruń Centre for Astronomy, Nicolaus Copernicus University, \\ ul. Gagarina 11, 87-100 Toruń, Poland, \\ email: aniedzi, grzenow, pawziel @astri.uni.torun.pl \\ ${ }^{2}$ Department of Astronomy and Astrophysics Pennsylvania State University, \\ 525 Davey Laboratory, University Park, PA 16802
}

\begin{abstract}
The main objective of the Penn State/Torun Centre for Astronomy search for planets around evolved stars is the detection of planetary systems around massive, evolved stars. We are also interested in the evolution of these systems on stellar evolution timescales. In this paper we present our approach to determine the basic physical parameters of our targets GK-giants. We also discuss the stellar activity indicators used in our survey: line bisector and curvature, and $\mathrm{H} \alpha$ variability.
\end{abstract}

Keywords. stars: fundamental parameters, stars: activity, (stars:) planetary systems

\section{Introduction}

Proper interpretation of the results from precision RV studies of GK-giants requires a detailed knowledge of their physical parameters. Effective temperatures and gravitational accelerations are needed to obtain luminosities, and, with the additional knowledge of metallicities, estimates of stellar masses and ages can be derived by means of the isochrone fitting. Together with estimates of stellar radii and rotation periods, these data allow one to address the influence of stellar surface inhomogeneities (spots) on the observed RV variations.

All alternative sources of RV variations in GK-giants have to be ruled out before substellar companion interpretation becomes acceptable. Unfortunately, the long period variations, if present, cannot usually be studied using data other than the existing photometry (usually of moderate quality), or the data collected from the RV survey. Therefore, the detailed activity discussion is usually based on the indicators defined on the basis of the same spectra as those used for the RV measurements. In this paper, we illustrate our methodology of the determination of stellar parameters and activity analysis based on the case of the K-giant PSU-TCfA 18, a potential planet hosting star.

\section{Observations}

Observations were made with the Hobby-Eberly Telescope (HET) and the High Resolution Spectrograph (HRS). The HRS was used in the $\mathrm{R}=60,000$ resolution mode with a gas cell $\left(I_{2}\right)$ inserted into the optical path, and it was fed with a 2 arcsec fiber. Typically, the signal-to-noise ratio per resolution element (at $594 \mathrm{~nm}$ ) was $\sim 200$ for the stellar spectra taken with the gas cell, and $\geqslant 250$ for the templates. 


\section{Basic stellar parameters}

The atmospheric parameters of the program stars were obtained with the spectroscopic method (Takeda et al. 2005a, b), which is based on analysis of Fe I and Fe II lines and relies on conditions resulting from the assumption of the LTE. Typically, over 200 FeI and about 25 FeII lines were measured for every star.

We tested a reliability of our determinations with the Takeda et al. (2005a) TGVIT code by applying it to 8 stars, for which the parameters have been published by Butler et al. (2006). A comparison of the results shows that $T_{\text {eff }}$ values agree to within $49 \mathrm{~K}$, $\log g$ to within 0.11 dex and $[\mathrm{Fe} / \mathrm{H}]$ to within 0.11 dex.

Stellar masses were derived by comparing the positions of stars in the HR diagram with the theoretical evolutionary tracks of Girardi et al. (2000) and Salasnich et al. (2000) for a given metallicity. For stars for which the parallax determinations are precise enough, the metallicity may introduce a significant uncertainty in mass because of the choice of an evolutionary track. We assume that for an average red giant with known parallax and good photometry the mass may be estimated to within $0.3 M_{\odot}$. The precision depends however on the actual star position in HRD. We also note that for stars in the red giant clump, which are in the fast evolution phase with mass-loss, the derived masses are probably the upper limits.

Stellar radii were determined using the calibration given in Alonso et al. (2000). Stellar ages are usually estimated with the aid of the theoretical stellar isochrones.

In the case of the PSU-TCfA 18 star, we have measured equivalent widths of up to 195 Fe I and 11 Fe II lines for further analysis. For this star, the basic physical parameters are $T_{\text {eff }}=4246 \mathrm{~K}, \log g=2.43, v_{t}=1.52 \mathrm{kms}^{-1},[\mathrm{Fe} / \mathrm{H}]=0.11, M=5.5 M_{\odot}$, and $R=$ $27.4 R_{\odot}$. Intrinsic uncertainties of our determinations are $\sigma T_{\text {eff }}=29 \mathrm{~K}, \sigma \log g=0.09$, $\sigma v_{t}=0.13 \mathrm{kms}^{-1}$ and $\sigma[\mathrm{Fe} / \mathrm{H}]=0.06$.

\section{4. $V \sin i$ measurements}

Rotation periods represent a parameter of particular importance in searches for planetary companions to red giants. As the rotation periods of these stars are very similar to expected orbital periods, their knowledge is critical for an unambiguous interpretation of observations. Any correlation of stellar activity indicators variations with the rotation period make a substellar companion hypothesis unlikely. To estimate rotation periods from our spectra we have used the cross-correlation technique, as described in Benz \& Mayor (1984).

We have computed the CCFs by cross correlating the high $\mathrm{S} / \mathrm{N}$ blue spectra with a numerical mask. To measure $V \sin i$ we have worked out a $V \sin i$ calibration for the HET/HRS. To determine the $\sigma_{0}$ vs. $(B-V)$ relationship, we have used 16 slow rotators with accurately known projected rotational velocities, preferably from Gray (1989), Fekel (1997) and de Medeiros \& Mayor (1999). For these stars we have determined $\sigma_{0}$ using the formula from Benz \& Mayor (1984) ( $\left.V \sin i=A \sqrt{\sigma_{o b s}^{2}-\sigma_{0}^{2}}\right)$ and assuming the constant $A=1.9$ following the Queloz et al. (1998) and Melo et al. (2001). We have carried out a least-squares fit to the data of the analytical function $\sigma_{0}=a_{2}(B-V)^{2}+a_{1}(B-V)+a_{0}$, which yields the following calibration: $\sigma_{0}=15.592(B-V)^{2}-26.753(B-V)+14.559$.

Using this calibration we have obtained $V \sin i=(3 \pm 1) \mathrm{kms}^{-1}$ for our star. Adopting the radius for this star as determined above, we have estimated its rotational period to be 220 - 950 days. The large uncertainty in the rotation period is caused by uncertainties related to the determination of the radius and $V \sin i$. 


\section{Stellar activity indicators}

One of possible sources of the observed RV variations in GK-giants is due to their pulsations. Therefore, photometric data that span long periods of time are needed for the interpretation of the results of our survey. Because we do not conduct our own parallel photometric observations, we must rely on the existing photometric databases like Hipparcos or NSVS (Woźniak et al. 2004). These moderate quality data provide time-series, which are long enough to be useful in searches for long-term periodicities. However, these measurements were usually performed many years prior to our RV survey. In the particular case of the PSU-TCFA 18 star, no detectable variability is present in the existing photometric data.

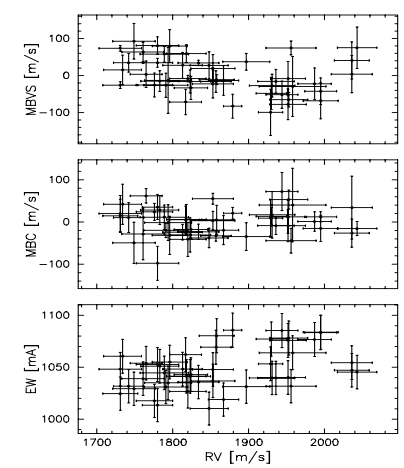

Figure 1. MBVS, MBC and EW of the $H \alpha$ for PSU-TCfA 18 as a function of radial velocity.

\subsection{Line bisectors}

The basic tool to study the origin of RV variations derived from the stellar spectra is the analysis of the shapes of spectral lines via line bisectors (Gray 1983).

We have computed line bisectors for 5 strong, unblended spectral features of a moderate intensity, which were located close to the center of echelle orders: Cr I $663.003 \mathrm{~nm}$, Ni I $664.638 \mathrm{~nm}$, Ca I $671.77 \mathrm{~nm}$, Fe I $675.02 \mathrm{~nm}$, and Ni I $676.784 \mathrm{~nm}$. All these lines show well defined bisectors.

The changes in the spectral line bisectors were quantified using the bisector velocity span $(B V S)$ parameter, which is simply the velocity difference between the upper and the lower points of the line bisector $\left(B V S=v_{3}-v_{2}\right)$, and the bisector curvature $(B C)$, which is the difference of the velocity span of the upper half of the bisector and its lower half $\left(B C=\left(v_{3}-v_{2}\right)-\left(v_{2}-v_{1}\right)\right)$. It is important to examine both $B V S$ and $B C$, because it is possible for a star to show variations in one of these parameters only. In choosing the span points, it is important to avoid the wings and cores of the spectral line, where the errors in the bisector measurements are large. For our span measurements we chose $v_{1}=0.29, v_{2}=0.57$, and $v_{3}=0.79$ in terms of the line depth at the line core. Using the bisector measurements of all 5 spectral lines we have computed the average velocity span and curvature after subtracting the mean value for each spectral line.

In Figure 1, we present the mean bisector velocity span $(M B V S)$ and the mean bisector curvature $(M B C)$ for our star, as a function of RV. Uncertainties in the derived values of $M B V S$ and $M B C$ were estimated as standard deviations of the mean. The correlation coefficients were found to be $r=-0.32 \pm 0.10$ for $M B V S$ and $r=0.07 \pm 0.02$ for $M B C$. It is clear that they are not correlated with radial velocities which supports the planetary mass companion hypothesis. The available RV measurements are not uniformly distributed over the estimated period, which is visible in Fig. 1 as a scatter varying with RV. More observations are needed to confirm the apparent lack of correlation. 


\subsection{H $\alpha$ variations}

Since our spectra start at $407.6 \mathrm{~nm}$ we cannot investigate the variation of the Ca II $\mathrm{K}$ emission line $(393.4 \mathrm{~nm})$. Also the infrared CaII triplet lines 849.8-854.2 are outside the range of our spectra. Therefore, we use $\mathrm{H} \alpha$ line $(656.28 \mathrm{~nm})$ as a chromospheric activity indicator. The EW measurements of the $H \alpha$ line can be made in our spectra with a typical precision of a few percent. To minimize contamination from telluric lines we measured the EW of the central part of the line profile defined by $\mathrm{I} / \mathrm{I}_{c} \leqslant 0.775$. In the case of our star, the measurements give a mean value of $1049 \pm 20 \mathrm{~m} \AA$. The rms value of $20 \mathrm{~m} \AA$ corresponds to $2 \%$ variation in the EW. In Figure 1 we present EW measurements for $H \alpha$ as a function of RV. The correlation coefficient of $r=0.44 \pm 0.10$ shows marginal relationship.

\section{Conclusions}

A detailed knowledge of stellar parametres of red giants is very important for interpretation of their RV variations. To rule out stellar activity as the source of such variations, one needs precise rotation periods and several other indicators to be measured at many epochs.

\section{Acknowledgements}

AN \& GN acknowledge the financial support from the MNiSW through grant 1P03D 007 30. GN is a recipient of a graduate stipend of the Chairman of the Polish Academy of Sciences. PZ was supported by MNiSW grant SPB 104E-3376. The Hobby-Eberly Telescope (HET) is a joint project of the University of Texas at Austin, the Pennsylvania State University, Stanford University, Ludwig-Maximilians-Universität München, and Georg-August-Universität Göttingen. The HET is named in honor of its principal benefactors, William P. Hobby and Robert E. Eberly.

\section{References}

Alonso, A., Salaris, M., Arribas, S., Martínez-Roger, C. \& Asensio Ramos, A. 2000, A $\&$ A, 355, 1060

Benz, W. \& Mayor, M. 1984, A\&A, 138, 183

Butler, R. P., Wright, J. T., Marcy, G. W., Fischer, D. A., et al. 2006, ApJ, 646, 505

Fekel, F. C. 1997, PASP, 109, 514

Fischer, D. A., \& Valenti, J. 2005, ApJ, 622, 1102

Frink, S., Mitchell, D. S., Quirrenbach, A., Fischer, D. A., Marcy, G. W., \& Butler, R. P. 2002, ApJ, 576, 478

Girardi, L., Bressan, A., Bertelli, G., \& Chiosi, C. 2000, A\&AS, 141,371

Gray, D. F. 1983, PASP, 95, 252

Gray, D. F. 1989, ApJ, 347, 1021

Hatzes, A. P., \& Cochran, W. D. 1993, ApJ, 413, 339

Hatzes, A. P., \& Cochran, W. D. 1994, ApJ, 422, 366

de Medeiros, J. R., \& Mayor, M. 1999, A\&ASS, 139, 433

Melo, C. F. H., Pasquini, L., \& de Medeiros, J. R. 2001, $A \mathscr{E} A, 375,851$

Queloz, et al. 1998, A\& A, 335, 183

Salasnich, B., Girardi, L., Weiss, A., Chiosi, C. 2000, A\&\&A, 361, 1023

Sato, B., Izumiura, H., Toyota, E., Kambe, E., Takeda, Y., et al. 2007, ApJ, 661, 527

Takeda, Y., Ohkubo, M., Sato, B., Kambe, E., \& Sadakane, K. 2005, PASJ, 57, 27

Takeda, Y., Sato, B., Kambe, E., Izumiura, H, Masuda, S. \& Ando, H. 2005, PASJ, 57, 109

Walker, G.A.H., Yang, S., Campbell, Bruce, I., \& Alan W. 1989, ApJ, 343, 21

Woźniak, P. R., et al. 2004, AJ, 127, 2436 